\title{
Polysilicate Aluminum Ferrite Coagulant Dyeing Wastewater Treatment Simulation and Computer Simulation Research
}

\author{
Juan Tan* \\ School of Civil Engineering and Architecture \\ University of Jinan \\ Jinan, 250022, China \\ E-mail: cea_tanj@ujn.edu.cn \\ *Corresponding author
}

\author{
Yanzhen $\mathrm{Yu}$ \\ College of Civil Engineering \\ Qilu Institute of Technology \\ Jinan, 250200, China \\ E-mail: cea_yuyz@ujn.edu.cn
}

\begin{abstract}
Fly ash, sodium carbonate, potassium hydrochloric acid solution and high-speed rail are the main raw material, prepared polysilicate aluminum ferrite coagulant (PSAF) and used for dyeing wastewater treatment simulation. This paper discusses homemade coagulation effect, at the same time, the introduction of an important parameter to characterize - floc fractal fractal dimension fractal structures from microscopic floc morphology studies floc angle between the macroscopic relationship with coagulation effect, is how to get the structure of dense settlement a good batting performance body, and it provides a research method. In addition, the application of computer simulation technology to build floc growth model, fractal floc formation under different coagulation conditions were simulated by comparing the two types of floc morphology and appearance of fractal dimension. This paper discusses the simulation model established in the coagulation test the feasibility of the application.
\end{abstract}

Keywords-coagulant flocculation, fractal dimension, simulation model

\section{INTRODUCTION}

Floc morphological structure on the edge of the internal largely determines the density of flocs and retrogradation, which affects the final water treatment effect. With the aid of microscope photos, Image $\mathrm{J}$ image processing software to detailed study from modern research and analysis method of coagulation reaction, floc growth process and the microstructure of the floc structure, it is different from the past can only from the macro according to the final coagulation effect of the removal rate of each index evaluation [1], the characterization of flocs fractal dimension of fractal flocs fractal features can be analysed quantitatively with the relationship between the coagulation effect [2], this paper, application of computer diffusion-limited condensed (group) model is established for different coagulation conditions the growth process of the flocs and morphological characteristics of simulation, by comparing the two types of the appearance of the floc morphology and the fractal dimension value, discusses the established simulation model in actual practicality in the study of coagulation, for how to obtain a compact structure, fast settling velocity of flocs and finding the best coagulation condition to provide a research method.

\section{EXPERIMENT}

\section{A. The main experimental equipment and software}

- KSMF2000 intelligent temperature control muffle furnace, SH05-3 electric magnetic stirrer.

- Olympus CX31 type biological microscope, Olympus 250 digital camera.

- MATLAB software, ImageJ image processing software.

\section{B. Determination of the fractal dimension of real floc}

It can be realized by image method in the determination of real fractal dimension of flocs, specific measure is: the completion of the index determination of coagulation experiment, draw a small amount of flocs and under microscope slides, the flocculant microscope photos for the real shape and figure, and USES the ImageJ software of flocs image processing to obtain the complete single flocs image, the operation is in the Image J software to open a flocs image (I) in Figure 1, with the "curve" toolbox surrounded the floc, and select "edit" - "clear" to get a single complete flocs (II) in Figure 1, in the heart of the "image" - "type" image is adjusted for the 8 bit greyscale (III) in figure 1, click the "adjust" - "threshold" and adjust the flocs is covered by the appropriate application (IV) in Figure 1, after clicking on the "magic wand" toolbox of flocs contour surrounded by bright yellow closed curve (Fig. 1V), select "analysis" - "measure" of flocs parameters such as area, perimeter P A calculated, and results pop-up window as shown in Figure 2, the area and perimeter $\mathrm{P}$ into $\mathrm{A}$ of flocs in Equation (1) to calculate the fractal dimension of flocs.

$$
D=\ln A / \ln P
$$

Where $\mathrm{A}$ is the area of the floc, $\mathrm{P}$ is the perimeter, $\mathrm{D}$ is the fractal dimension.
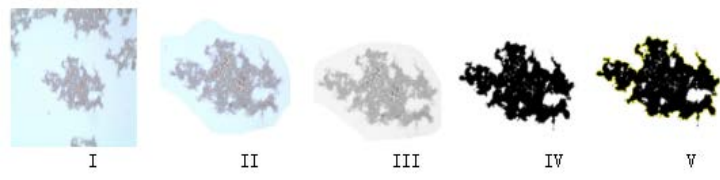

Figure1. ImageJ software processing flocs image sketch 


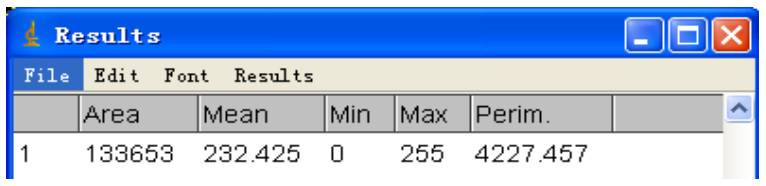

Figure2. Floc area, perimeter calculation results

\section{Correlation between the fractal dimension and the coagulation effect of flocs}

By introducing the fractal dimension of the parameters, it can characterize the fractal flocs fractal characteristics of internal structure, and explore the real floc irregular filling degree and the edge height from the micro point of view, the real shape and structure of floc coagulating reaction after detailed research, through the investigation of flocs fractal dimension with various relationships between removal rate of mixed coagulation under the condition, the study of the correlation between them.

1) The correlation between the removal rate and the fractal dimension of the different dosage of Chromaticity

Through the correlation relationship between morphology and structure of floc body study investigating different dosage on color removal and the rate of the flocs fractal dimension with the final coagulation effect, shown in Figure 3.

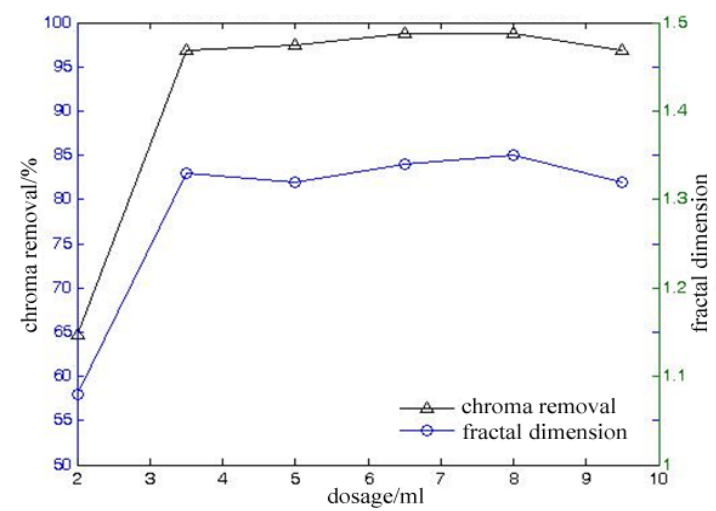

Figure 3. Under different dosing quantity Chroma removal rate and the correlation of fractal dimension

Figure 3 shows the results of the minimum dosage, the smaller fractal dimension of floc was only 1.08, at this time the floc structure loose easy to settlement, and Chroma removal rate is low, when the dosage was increased to $3.5 \mathrm{ml} / \mathrm{L}$ when the fractal dimension increases to 1.33 , floc structure became dense conducive to settlement, the Chroma removal rate increased, and the dosage increased to maintain the basic fractal dimension is larger and the same color removal rate of change is similar, from the overall change in two curve situation is similar to the dosage Chroma removal rate and fractal dimension of floc change trend.

2) The correlation between the removal rate and the fractal dimension of the color with different $\mathrm{pH}$ values

After the effects of $\mathrm{pH}$ value of the relationship between Chroma removal rate and the floc fractal dimension, in the form of floc structure with the final coagulation effect correlation, shown in Figure 4.

Figure 4 shows that $\mathrm{pH}=6.0$ when the flocs fractal dimension is 0.91 , the formation of the floc structure is loose, sedimentation performance is poorer, Chroma removal rate is low, fractal dimension increases gradually with the increase of $\mathrm{pH}$ and highest in the removal rate of Chroma $(\mathrm{pH}=7.5)$ reached the maximum value of 1.38 , and continue to increase the $\mathrm{pH}$ fractal dimension is reduced gradually change, also became loose, floc sedimentation velocity slowing, thus appeared the Chroma removal rate decreases, the two curves reflect the changing trends of the Chroma removal rate under different $\mathrm{pH}$ and the fractal dimension of flocs have a good correlation.

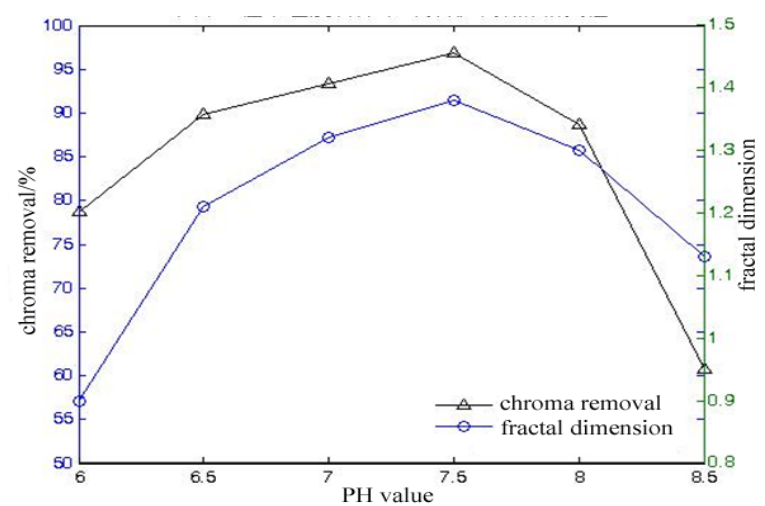

Figure 4.Under different $\mathrm{pH}$ Chroma removal rate and the correlation of fractal dimension

\section{SIMULATION OF FLOC}

\section{A. The establishment of simulation model}

Based on the computer simulation technique and mathematical modeling as the foundation, the establishment of diffusion limited aggregation (Group) condensation growth process model to simulate the mixed particle coagulation experiment in motion, collision, coagulation and floc fractal structure.

1) Diffusion limited cohesion model (DLA model) [3]

DLA growth model is based on a center particle as the center, around the disorderly attached to a certain number of particles, forming a condensed matter has obvious geometric center, condensed matter has self-similarity and scale invariance of morphological characteristics, the morphological structure of condensed matter can simulate water treatment in the complex fractal structure of flocs.

2) Diffusion limited cohesion (group) model (DLCA model) [4] [8]

DLCA growth model is within the limited space to as all particles or group can make the seed of the random walk, at the same time when the collision between particles or groups to condense into a new group with each other and swimming as condensed matter continues to spread, further condensed with collision of particles or other condensed matter, finally, all the particles or group into a no obvious geometry center and a significant fractal structure of condensed matter.

\section{B. Simulation of real flocs}

In the premise of obtaining the real flocs topography and fractal dimension of floc, real simulation model of floc growth through selecting proper and suitable parameters.

The main idea of simulation real flocs is made in the appearance of real flocs and simulation flocs graph similarity, also make the two fractal dimension equal characterization of two kinds of fractal flocs or similar. 
1) Simulation of different dosage on real flocs

In after completion of the index determination of Figure 3 experiment for the dosing quantity of flocs under micro topography and corresponding fractal dimension, the selection of appropriate DLA model or DLCA model and conform to the parameters on the simulation, find appropriate simulation, simulation results as shown in Figure 5.
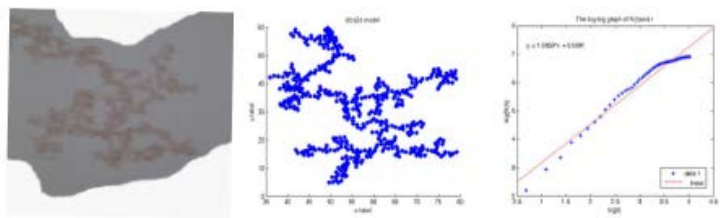

$2.0 \mathrm{~mL}$
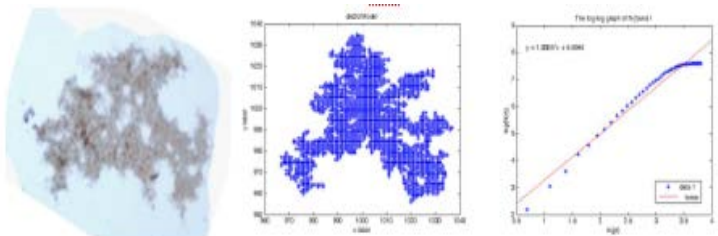

$3.5 \mathrm{~mL}$
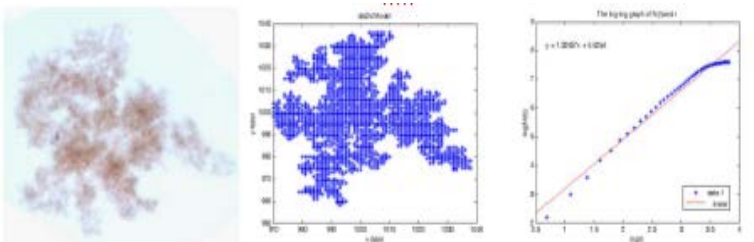

$5.0 \mathrm{~mL}$
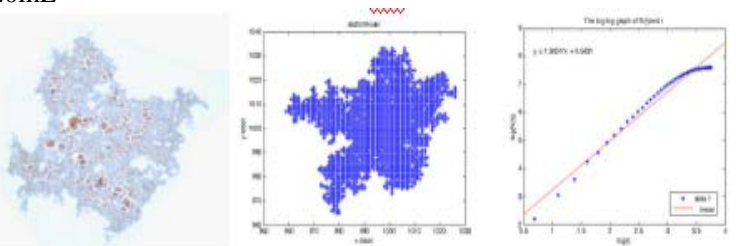

$6.5 \mathrm{~mL}$
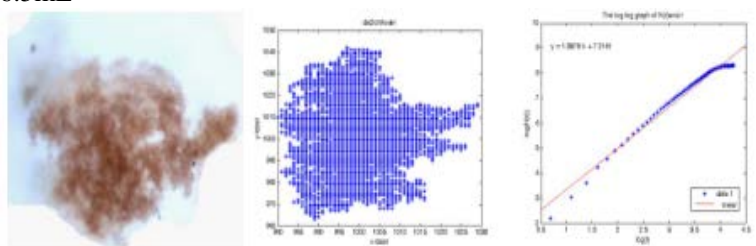

$8.0 \mathrm{~mL}$
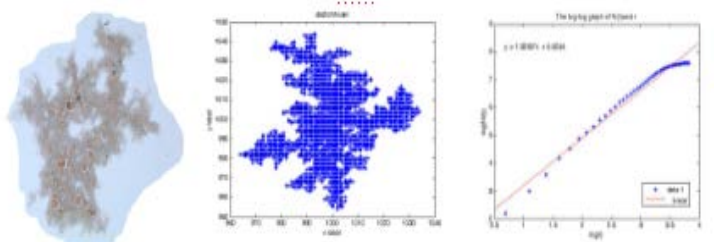

$9.5 \mathrm{~mL}$

Figure 5. Simulation real flocs results under different dosing quantity

Through real flocs in Fig.5, compared with the appearance of the simulation figure floc morphology, it is not difficult to find that high similarity of the two, at the same time by Figure 3 under the result, the additive amount of real fractal dimension of flocs were 1.08, 1.33, 1.32, 1.34, 1.35, 1.32, respectively, and corresponding simulation in figure 5 fractal dimension of flocs were about 1.09, 1.33, 1.33, 1.09, $1.36,1.33$, and the fractal dimension is basically equal or close to, in addition also found in dosing quantity smaller if $2.0 \mathrm{ml} / \mathrm{L}$, the floc structure is loose and the fractal dimension is small, at this time is suitable for the DLCA model simulation, and dosing quantity increases to $3.5 \mathrm{ml} / \mathrm{L}$, floc sedimentation effect is good, its fractal dimension also increases to 1.33, the DLA should be adopted model for simulation, after that with the increase of additive amount of real floc structure remains good settleability, fractal dimension and basic are of higher value, should the DLA model to simulation, experiments show that the established simulation model of the dosing quantity under real simulation result has a good effect of flocs.

2) Simulation of different $\mathrm{pH}$ on real flocs

According to the $\mathrm{pH}$ value of flocs figure and corresponding fractal dimension, select appropriate models and parameters on the simulation, the simulation results as shown in Figure 6.
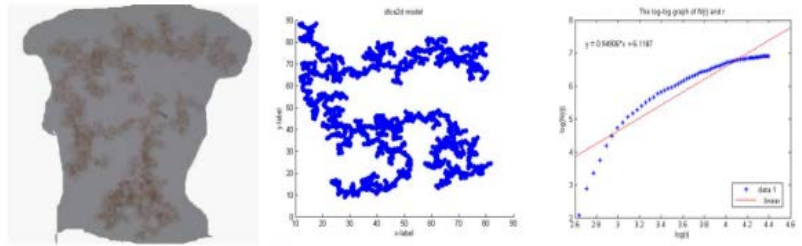

$\mathrm{pH}=6.0$
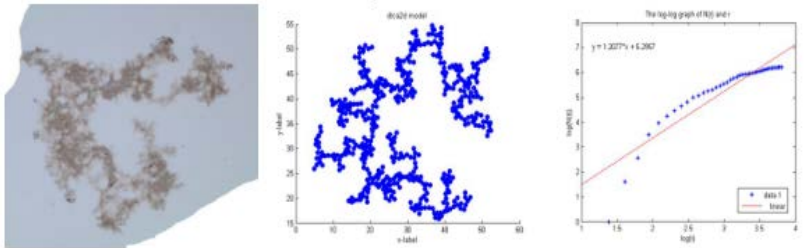

$\mathrm{pH}=6.5$
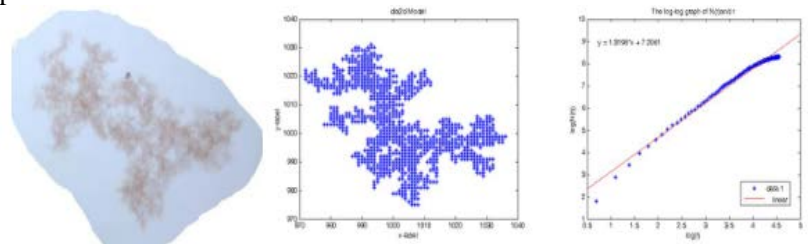

$\mathrm{pH}=7.0$
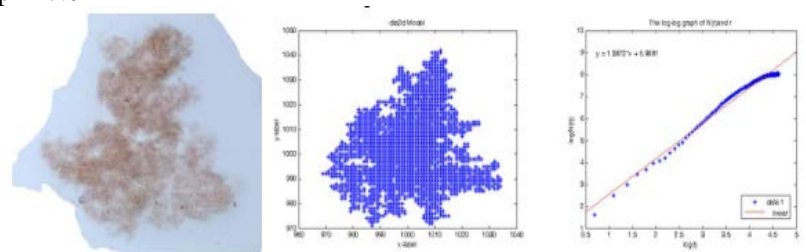

$\mathrm{pH}=7.5$
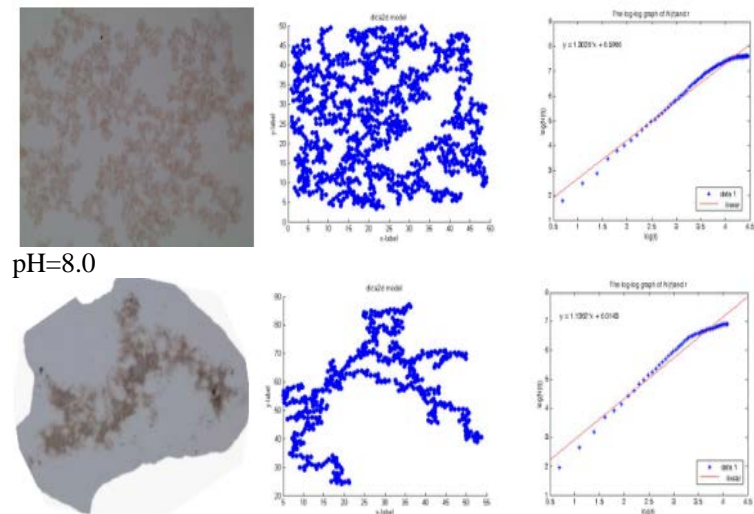

$\mathrm{pH}=8.5$

Figure 6. Simulation real flocs results under different $\mathrm{pH}$

In Figure 6, compared two types of floc configuration diagrams, it has higher similarity in appearance shape, Figure 4 result under different $\mathrm{pH}$ real fractal dimension of flocs were $0.91,1.22,1.32,1.38,1.30,1.13$, respectively, and simulate the fractal dimension is about $0.95,1.21,1.32,1.39$, 
$1.30,1.39$, both the size of the content is equal or similar principle, also found that only when the $\mathrm{pH}=7.5$ the densest floc structure, the fastest sedimentation velocity (that is, the fractal dimension maximum) to fit the DLA model for the simulation, $\mathrm{pH}$ value, the other should be the DLCA model simulation, the experiment also shows that the established under different $\mathrm{pH}$ value of the real simulation result has a good effect of flocs.

In summary, the established two simulation models for different coagulation floc real flocs under the conditions of simulated with good results.

\section{RESULTS}

In characterization of flocs fractal dimension and fractal form, from the microstructure of floc reaction water treatment effect, explores the real structure of the flocs with the intrinsic relationship between coagulation effect, in the coagulation floc morphology and coagulation effect of set up a bridge of theory with practice, to the actual coagulation experiment provides a new and useful research methods, the coagulation effect and the correlation of fractal dimension of flocs experiment results show that the coagulation under different conditions of coagulation effect and between the fractal dimension of flocs with good correlation.

Simulation model based on operation performance and visual computer, MATLAB software powerful, can the past cannot be detailed research of disordered coagulation reaction process of chaos unfolded on the micro visual form, for in-depth knowledge of mixed colloids and suspended particle coagulation in the solution without regular and random swimming, collision, coagulation and flocculation body growth process and fractal structure provides an effective research approach, the final simulation results also show that the establishment of two floc simulation model has a strong practicality and reliability in water treatment.

\section{ACKNOWLEDGMENT}

This work was funded by A Project of Shandong Province Higher Educational Science and Technology Program (Nos. J17KA200).

\section{REFERENCES}

[1] Arman Vahedi, Beata Gorczyca, "Application of fractal dimensions to study the structure of flocs formed in line softening process”, Water Research, Vol.45, pp.545-556,2011.

[2] M. M. Fernández-Martínez,M.A. Sánchez-Granero, "Fractal dimension for fractal structures”,Topology and its Applications, Vol.163,pp. 93-111,2013.

[3] Mandelbrot B B, Fractal objects-shape, chance and dimension. Hong Su Translated. Beijing: World Book Publishing Company, 1999.

[4] Jin PK,Wang XH,Guo K, "DLA simulation of fractal flocs andcalculation of fractal dimension”,Enviromental Chemistry, Vol.26(1),pp.4-9,2007.

[5] Tang P, Greenwood J, Raper J A,“A model to describe the settling behavior of fractal aggregates”, J Colloid Interface Sci,Vol. 247(1),pp.210-219,2002.

[6] Jan Andres, Miroslay Rypka,"Self-similar fractals with a given dimension and the application to quantitative linguistics”, Nonlinear Analysis: Real World Applications, Vol.13,pp.42-53,2012.

[7] N.S. Lam,“Fractal Analysis”,International Encyclopedia of Human Geography, Vol.13,pp.263-270,2009.

[8] Jr-Lin Lin, Chihpin Huang,"Coagulation dynamics of fractal flocs induced by enmeshment and electrostatic patch mechanisms”, Water Research, Vol.42,pp.4457-4466,2008. 